\title{
Modeling of Measured Target Pressure Profiles in Three Hypervelocity Impact Experiments
}

\author{
M. Gerassimenko
}

This article was submitted to the

Hypervelocity Impact Symposium 2000, Galveston, TX, November $6-10,2000$ 


\section{DISCLAIMER}

This document was prepared as an account of work sponsored by an agency of the United States Government. Neither the United States Government nor the University of California nor any of their employees, makes any warranty, express or implied, or assumes any legal liability or responsibility for the accuracy, completeness, or usefulness of any information, apparatus, product, or process disclosed, or represents that its use would not infringe privately owned rights. Reference herein to any specific commercial product, process, or service by trade name, trademark, manufacturer, or otherwise, does not necessarily constitute or imply its endorsement, recommendation, or favoring by the United States Government or the University of California. The views and opinions of authors expressed herein do not necessarily state or reflect those of the United States Government or the University of California, and shall not be used for advertising or product endorsement purposes.

This is a preprint of a paper intended for publication in a journal or proceedings. Since changes may be made before publication, this preprint is made available with the understanding that it will not be cited or reproduced without the permission of the author.

This report has been reproduced directly from the best available copy.

Available to DOE and DOE contractors from the Office of Scientific and Technical Information

P.O. Box 62, Oak Ridge, TN 37831

Prices available from (423) 576-8401

http://apollo.osti.gov/bridge/

Available to the public from the

National Technical Information Service

U.S. Department of Commerce

5285 Port Royal Rd.,

Springfield, VA 22161

http://www.ntis.gov/

OR

Lawrence Livermore National Laboratory

Technical Information Department's Digital Library

http://www.llnl.gov/tid/Library.html 


\title{
MODELING OF MEASURED TARGET PRESSURE PROFILES IN THREE HYPERVELOCITY IMPACT EXPERIMENTS
}

\author{
MICHEL GERASSIMENKO
}

\author{
Lawrence Livermore National Laboratory \\ 7000 East Avenue, Livermore, CA 94550
}

\begin{abstract}
A $24 \mathrm{~g}$ aluminum sphere was shot at a sparse array of cylinders with nominal initial projectile velocity of 4 and $5 \mathrm{~km} / \mathrm{s}$. Pressure profiles were measured with cased carbon resistor gages at two locations in a projectile impacted water filled cylinder and two of its neighbors on three shots. The pressure maxima were in the $1-13$ kbars range. The experiments are modeled with the ALE3D code and several techniques are used to concentrate zoning at places of interest. There is excellent agreement between the measured and calculated pressure profiles for two shots and good agreement for the third. Comparison of the calculated pressure profiles with those from more refined calculations for two shots suggest that we are near convergence with respect to zone size.
\end{abstract}

Keywords: hypervelocity impacts, pressure measurements, pressure modeling.

\section{INTRODUCTION}

Experiments have been carried out under the PLATE program funded by the BMDO to measure quantities that can be compared directly with modeling calculations. The notion was to use a sphere to impact a water filled cylinder. Three more water filled cylinders and two solid ones were placed in close proximity to the impacted one. Three pressure gages were placed in each water filled cylinder. An artist's representation of the experimental setup is shown in Figure 1. Pressure profiles were obtained in three of the four water filled cylinders on three shots. In this report we compare the pressure profiles measured on shots 9714,9715 , and 9716 with modeling calculations performed with the ALE3D code.

\section{MEASUREMENTS}

The projectile was a $2.54 \mathrm{~cm}$ diameter $7075 \mathrm{~T} 651$ aluminum sphere moving along a direction normal to the axis of the cylinders. The water filled cylinders were made of 316 stainless steel, with an outside diameter of $6.35 \mathrm{~cm}$ and an inside one of $5.08 \mathrm{~cm}$. Separation between the cylinder walls was equal to the wall thickness, i.e., $0.635 \mathrm{~cm}$. The nominal projectile hitpoint was squarely on the front of the water filled cylinder with the projectile center in a plane normal to the cylinder axis, containing the sensing element of one of the pressure gages placed in the cylinders. The actual locations of the hitpoints and measured projectile velocities are given in Table 1. 


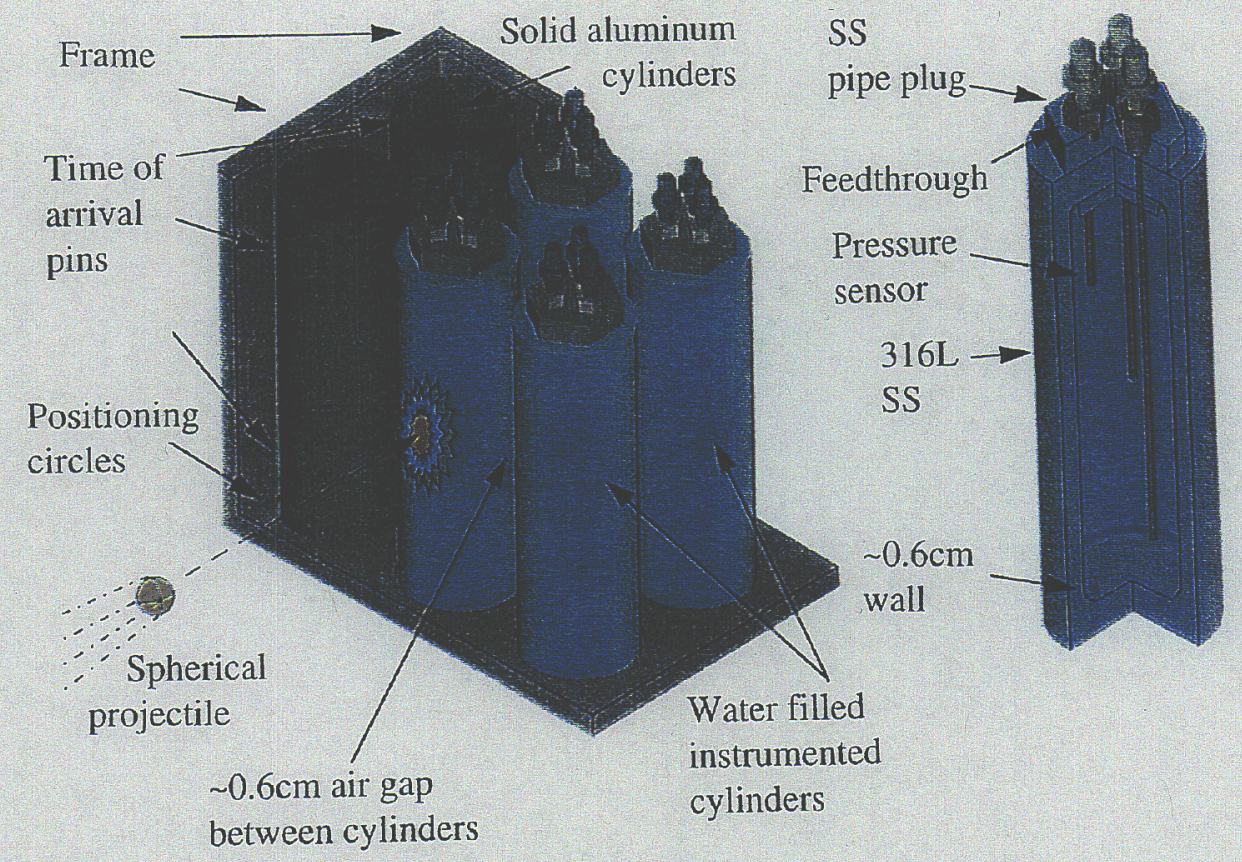

Fig. 1. Artist's view of the experiments and cutaway view of an instrumented water filled cylinder showing the location of the pressure sensors.

Table 1. Test Conditions

\begin{tabular}{|l|c|c|c|}
\hline Shot & 9714 & 9715 & 9716 \\
\hline Projectile & \multicolumn{3}{|c|}{$2.54 \mathrm{~cm}$ diameter aluminum sphere } \\
\hline Velocity & $4.09 \mathrm{~km} / \mathrm{s}$ & $4.03 \mathrm{~km} / \mathrm{s}$ & $4.92 \mathrm{~km} / \mathrm{sec}$ \\
\hline Aimpoint & \multicolumn{3}{|c|}{$\begin{array}{c}\text { Cylinder centerline in horizontal plane } \\
11.27 \mathrm{~cm} \text { from cylinder bottom }\end{array}$} \\
\hline $\begin{array}{l}\text { Actual } \\
\text { shotline }\end{array}$ & $\begin{array}{c}0.76 \mathrm{~cm} \text { right } \\
1.65 \mathrm{~cm} \text { low }\end{array}$ & $\begin{array}{l}0.76 \mathrm{~cm} \text { right } \\
0.00 \mathrm{~cm} \text { high/low }\end{array}$ & $\begin{array}{l}0.13 \mathrm{~cm} \text { right } \\
0.32 \mathrm{~cm} \text { high }\end{array}$ \\
\hline
\end{tabular}

The pressure sensors were manufactured by Dynasen, Inc., which refers to them as pinducers. They consist of a $50 \Omega$ carbon composition resistor, which is a $1 / 4$ watt $100 \Omega$ resistor cut in half, encased in a $3 \mathrm{~mm}$ wide brass tube. The sawed off end of the resistor is electrically tied to the brass tube, the other end is a wire, which is tied to the center conductor of a feedthrough that brings the sensor signal outside the water filled cylinder. The feedthrough assembly at the top of each water filled cylinder was designed to fail at a pressure exceeding that which causes rupture of the cylinder wall. In the experiments all feedthrough assemblies survived even though all impacted cylinders were ruptured and all adjacent cylinders were perforated. Mounting considerations made us position the pinducers on a $2.22 \mathrm{~cm}$ diameter virtual cylinder coaxial with the water filled cylinder. The pinducer locations are depicted in the right portion of Figure 1. One pinducer is located in the plane normal to the cylinder axis containing the nominal 
projectile hitpoint, $1.1 \mathrm{~cm}$ further away from the projectile hitpoint than the cylinder axis. We shall refer to it as the middle pinducer. Another is located in a plane parallel to the one described above but $5.08 \mathrm{~cm}$ higher and the pinducer positioned $120^{\circ}$ counterclockwise, as viewed from above, from the middle pinducer on the $2.22 \mathrm{~cm}$ diameter circle. We shall refer to it as the high pinducer. The third pinducer is located in a plane $5.56 \mathrm{~cm}$ lower, its position $120^{\circ}$ clockwise, as viewed from above, from the middle pinducer. We shall refer to it as the low pinducer. Referring back to Figure 1, the water filled cylinder to the right of the impacted one is the adjacent cylinder, the one behind the impacted cylinder is the rear cylinder, the fourth water filled one the diagonal cylinder.

Good signals were obtained from the middle and high pinducers. The low pinducer brass tube was subjected to high pressure before a signal was present at the sensor location, so only a couple of meaningful profiles were obtained. Pinducers in the impacted cylinder produced only short records lasting 5-10 $\mu \mathrm{sec}$ for the middle one and 10-29 $\mu \mathrm{sec}$ for the high one before pinducer failure due to high pressure. Pinducers in the diagonal cylinder produced either flat records or ones with baseline shifts with only a couple of exceptions.

A thorough pinducer calibration conducted by Dynasen proved to be substantially different from the nominal calibration originally published [1]. We believe this difference is due in large part to the mounting of the pinducer during calibration. The nominal and new calibrations are shown in Figure 2.

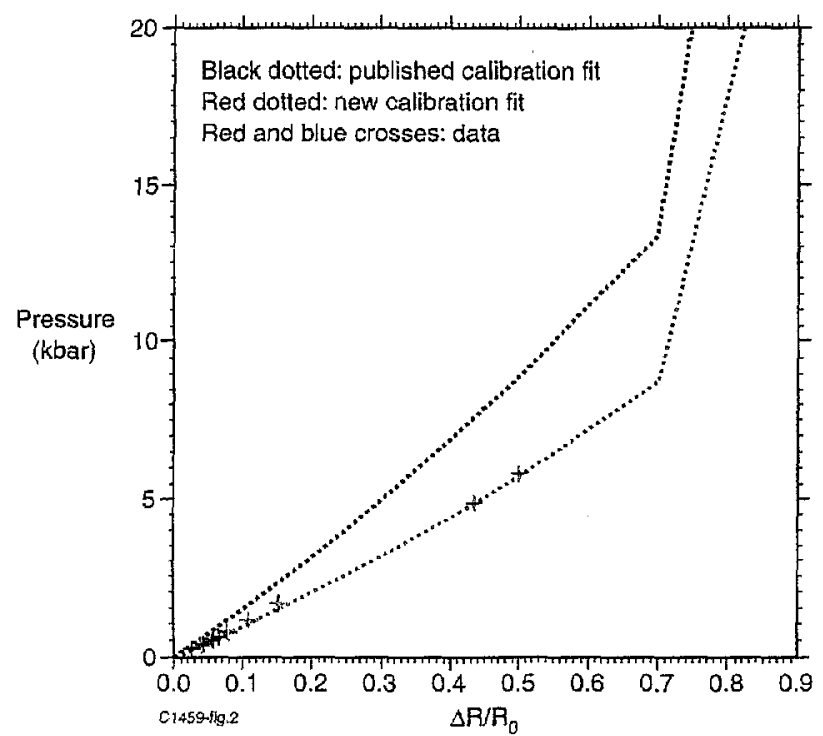

Fig. 2. New calibration of pressure sensors from the same batch as those used in the shots. In the 0.2-1.7 kbar range two sensors were calibrated, the data are indicated as red and blue crosses. The dotted line is a fit to the data scaled from the published sensor response [1]. 
The impacted cylinder pressure profiles go to a readout system saturation high pressure when the pinducer is broken by the shock. A final caveat is that pinducers, like most pressure sensors, are not well characterized in very fast pressure release. Carbon composition resistors used as pressure gages have been reported to respond more slowly to pressure decrease than to pressure increase $[2,3]$.

\section{MODELING}

The shots are modeled with the ALE3D code. The background material is air at STP even though the tests were carried out in an initially evacuated chamber. Given the small distance the projectile travels before impact and the close proximity of the water filled cylinders, this should have minimal impact on our results. Gruneisen equations-of-state are used for all materials except air: 7075-T6 aluminum for the projectile, 304 stainless steel for the water filled cylinders, 2024-T4 aluminum for the solid cylinders. The water filled cylinders are made of annealed 316L stainless steel, which has a similar yield stress to 304 stainless steel. A Steinberg-Guinan constitutive model is used for the metals. Failure in tension is set at $8 \mathrm{kbars}$ for the projectile, $30 \mathrm{kbars}$ for the steel and at an effective plastic strain of 0.2 for the steel. Water can support only 10 millibars in tension. Pressure equilibration of materials in mixed zones is turned off. The dimension of the pinducer is similar to the zone size in the water; we, therefore, do not model the pinducer but put a tracer point at its location. The calculated pressures are a weighted (by distance) average of all zones adjacent to, and including, the one containing the tracer point.

ALE3D allows one to concentrate zoning in areas of interest. We start out with specialized zoning concentrated in the impacted and adjacent cylinder. Material weights keep zoning concentrated in these cylinder walls and the projectile during the calculation. A cross-section through the projectile midplane normal to the cylinder axes (plane A) at initial setup and after relaxation prior to the start of the calculation is shown at the top in Figure 3. Weighting based on effective plastic strain keeps zoning concentrated on the projectile and its fragments, some of which impact the adjacent cylinder. The bottom of the figure is another plane A cross section, but at a later time, which illustrates this point. Since the rear cylinder is barely penetrated, relaxation in its wall is not allowed until the effective plastic strain reaches 80 percent of the value that produces failure. The same condition is imposed on the diagonal cylinder.

We have adjusted the experimental timing so that the first pressure rise seen by the middle pinducer in the impacted cylinder is simultaneous with the calculated one. This is done because experimental timing uncertainty appears to be several microseconds. It must be emphasized that this adjustment does not change the relative timing of the pressure peaks in each cylinder. After pinducer failure, usually indicated by a saturated high-pressure reading, the calculated profile is shown as a dashed line. Comparison of the measured and calculated profiles is made difficult by the large range in pressure. To alleviate this, we have multiplied the adjacent and rear cylinder pressures by constant factors to make their amplitude more comparable to that in the impacted cylinder. 

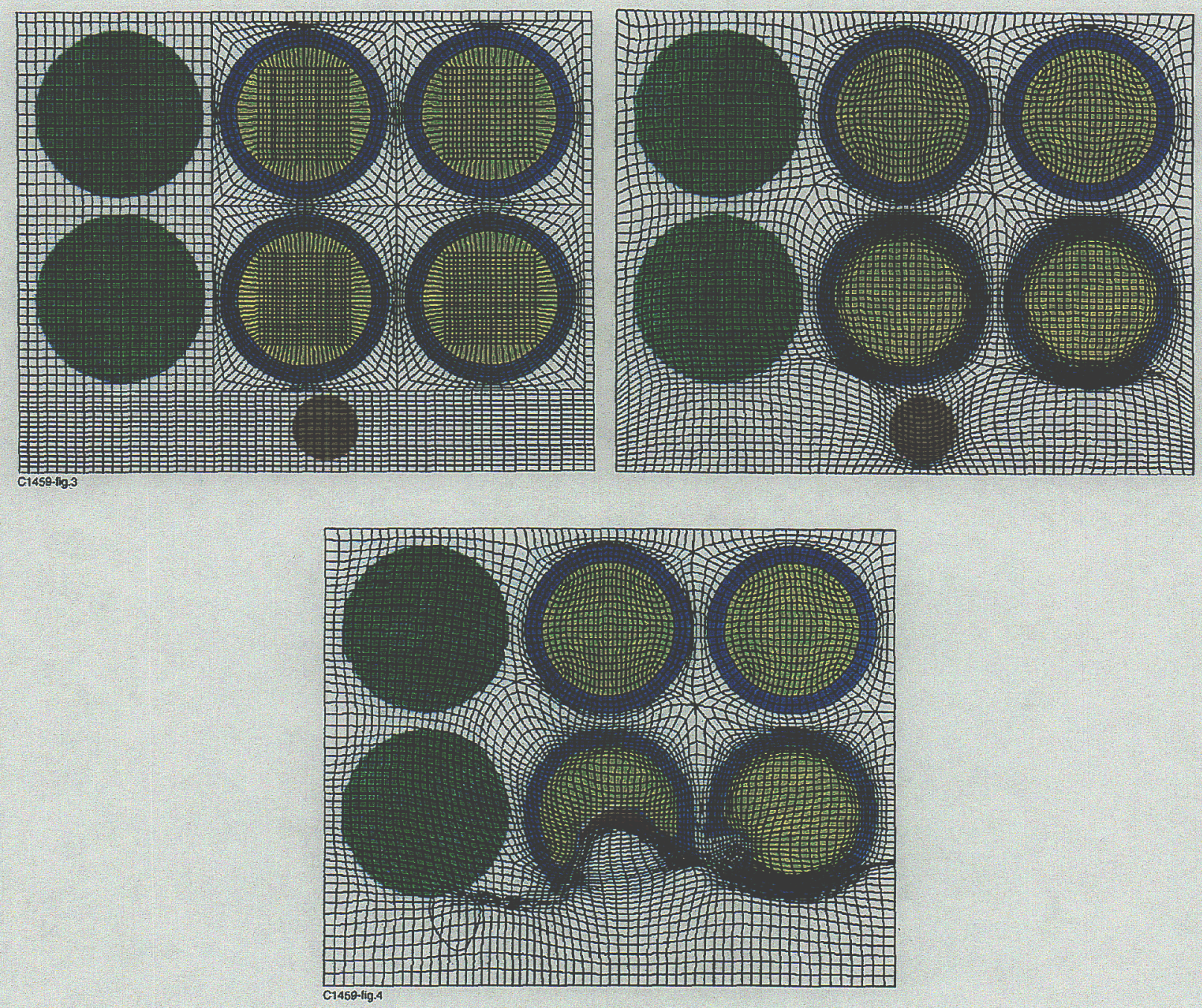

Fig. 3. Cross-sections through the projectile midplane normal to the cylinder axes with materials and zoning indicated. Top left: original problem setup. Top right: problem start after relaxation of the original problem setup everywhere except the walls of the rear and diagonal cylinders. Bottom: weighting based on effective plastic strain produces refined zoning where projectile deformation and break-up is occurring $20 \mu \mathrm{s}$ into the calculations.

A couple of points must be kept in mind as we compare the calculated profiles to the measured ones. The calculated profiles are much more rounded than the measured ones. This is due to several factors: zone size, artificial viscosity (which spreads to shock over several zones) and weighted averaging of the pressure over all zones adjacent to the one containing the tracer point and including it. This last factor is used to prevent large jumps in the calculated pressure as the tracer point crosses a zone boundary. The overall effect of these factors is to smooth the pressure profile, increase its rise time and reduce its peak in inverse proportion to its width i.e., by a little bit for a broad pressure pulse and by more for a narrow one. 


\section{Shot 9714}

The calculated and measured pressure profiles for the middle and high pinducers are shown in Figure 4. For the middle pinducer the agreement between the calculated and measured profiles is excellent. The adjacent cylinder has little water left after the first pressure pulse, so both the data and calculations may well be unreliable after about 60-70 $\mu \mathrm{sec}$. For the high pinducer the agreement between the calculated and measured profiles is very good. The adjacent cylinder calculated profile lags a bit at early times relative to the measurements. The rear cylinder profile amplitude is low, but only by about $20 \%$ at the peak. Overall the agreement between measured and calculated pressures is remarkable for shot 9714.
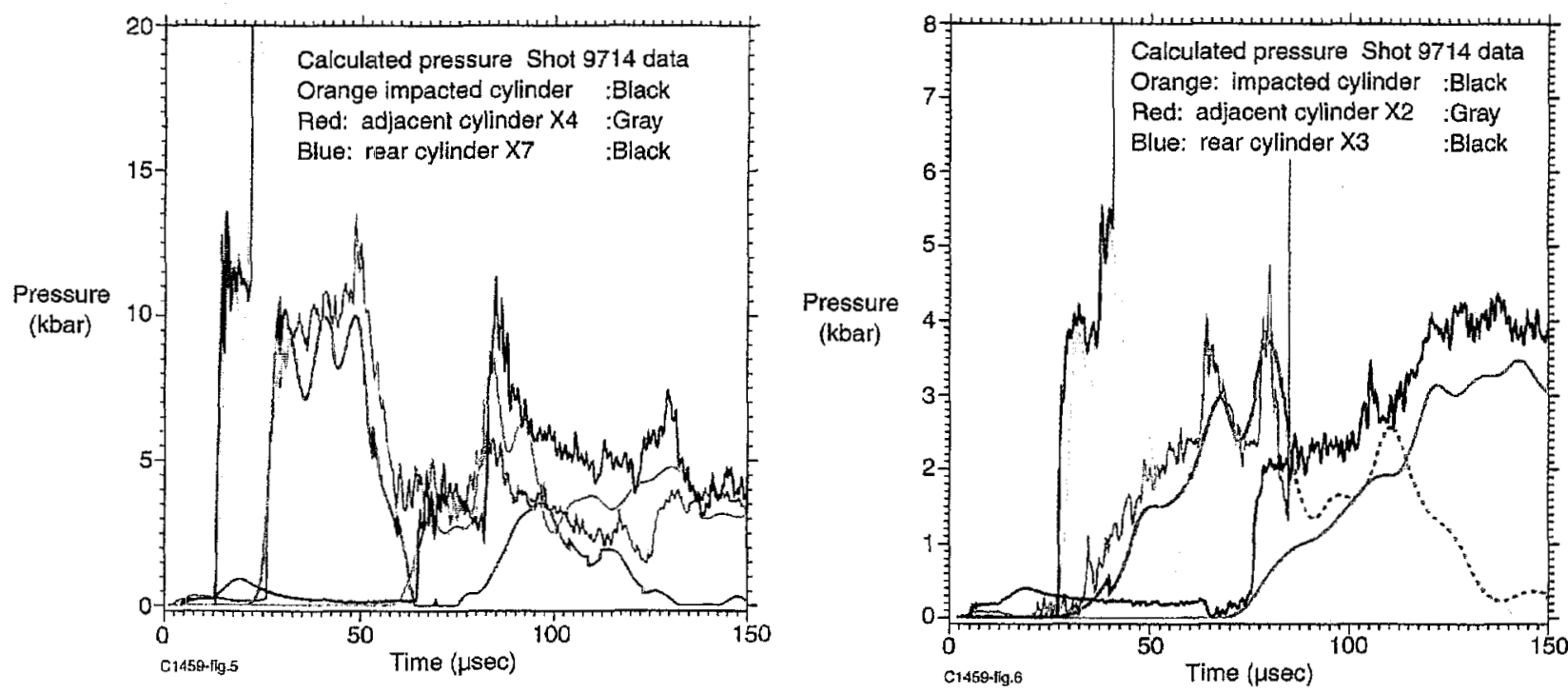

Fig. 4. Calculated and measured middle pinducer pressure profiles for shot 9714. Left: middle pinducer. Right: high pinducer. The adjacent and rear cylinder profiles have been multiplied by constant factors to make their amplitude more comparable to that from the impacted cylinder. The calculated profiles are dotted after pinducer failure.

\section{Shot 9715}

The calculated and measured pressure profiles for the middle and high pinducers are shown in Figure 5. For the middle pinducer the agreement between the calculated and measured profiles is very good. Timing of the calculated rear cylinder profile is a few microseconds early. The amplitude of the calculated impacted cylinder pressure is higher than the measurements, but this is at pressures near $8 \mathrm{kbars}$ where the slope of the fractional resistance change vs. pressure curve increases, so the measured pressure is uncertain on the high end. For the high pinducer the agreement between the calculated and measured profiles is good. It actually is excellent, except for the late timing of the calculated adjacent cylinder pressure. This timing is quite sensitive to the vertical location of the impact point. Overall the agreement between measured and calculated pressures is remarkable for shot 9715 . 

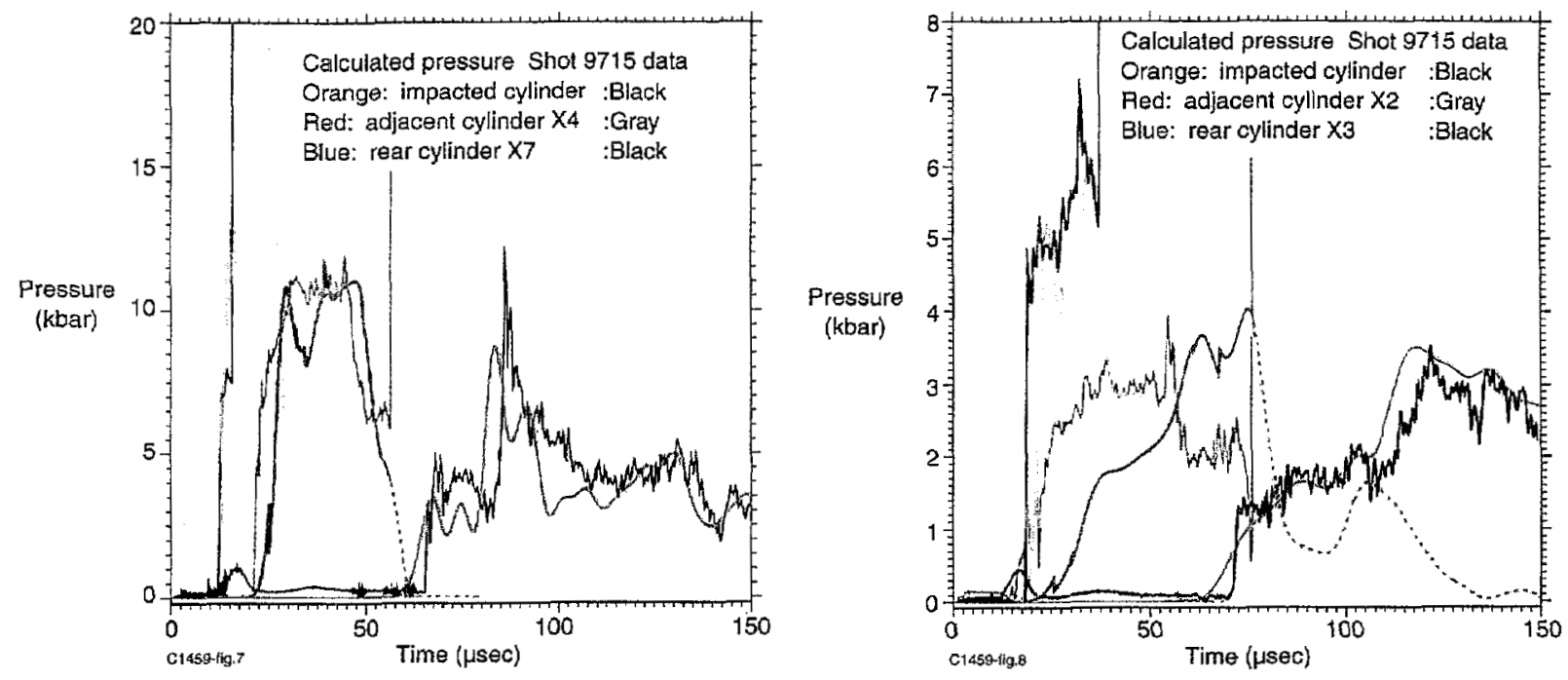

Fig. 5. Calculated and measured pressure profiles for shot 9715. Left: middle pinducer. Right: high pinducer. The adjacent and rear cylinder profiles have been multiplied by constant factors to make their amplitude more comparable to that from the impacted cylinder. The calculated profiles are dotted after pinducer failure.

\section{Shot 9716}

The calculated and measured pressure profiles for the middle and high pinducers are shown in Figure 6. For the middle pinducer the agreement between the calculated and measured profiles is excellent for the impacted cylinder, very good for the adjacent cylinder and good in amplitude but off by nearly $10 \mu \mathrm{sec}$ in timing for the rear cylinder. For the high pinducer the agreement between the calculated and measured profiles is very good in amplitude in all three cases, but the timing of the calculated profile is about $3 \mu \mathrm{sec}$ early for the impacted cylinder and $10-15 \mu \mathrm{sec}$
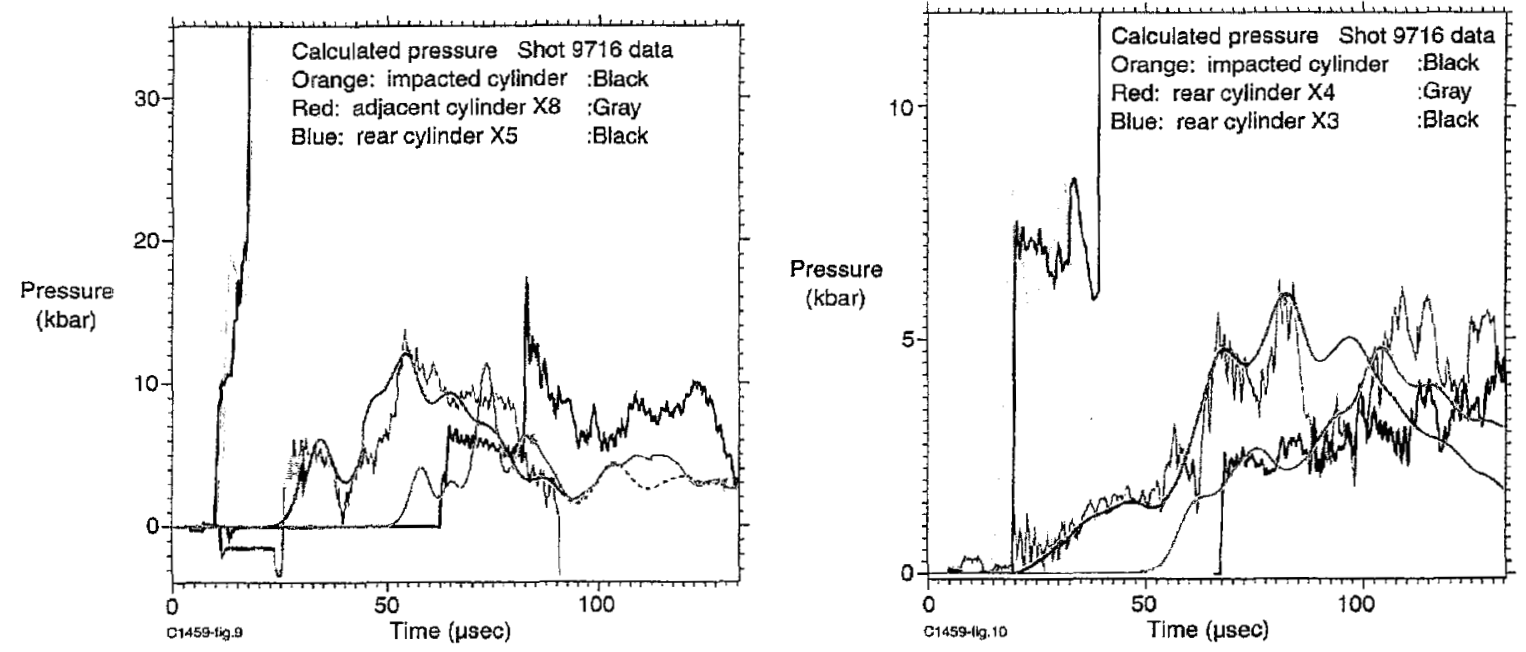

Fig. 6. Calculated and measured pressure profiles for shot 9716. Left: middle pinducer. Right: high pinducer. The adjacent and rear cylinder profiles have been multiplied by constant factors to make their amplitude more comparable to that from the impacted cylinder. The calculated profiles are dotted after pinducer failure. 
early for the rear one. Overall the agreement between measured and calculated pressures is good, marred only by some timing effects, for shot 9716 .

\section{CONVERGENCE OF THE PRESSURE PROFIL CALCULATIONS}

The ALE3D calculations that modeled the pressure profiles on shots 9714,9715 , and 9716 used $\sim 340 \mathrm{k}$ zones with an average zonal dimension of $0.286 \mathrm{~cm}$, but with zoning concentrated where deemed appropriate. While these baseline calculations produce pressure profiles quite close to the measured ones, we need to consider whether these profiles have converged with respect to zone size.

It is impractical to run calculations much larger than $340 \mathrm{k}$ zones, but we did run a couple, which used a symmetry plane and had an average zone size of $0.211 \mathrm{~cm}$. These calculations had a spacing between the cylinder walls of $0.60 \mathrm{~cm}$, while the baseline calculations used in this report have a spacing of $0.64 \mathrm{~cm}$ which is the specified distance in the tests. This small difference produces a small but finite difference in the calculated pressure profiles. So as not to confuse the effect of this small difference in cylinder spacing with that due to zoning, we compare the symmetry plane calculations with calculations identical to our baseline calculation except for the spacing between cylinder walls which is set at $0.60 \mathrm{~cm}$. The pressure profiles at the middle pinducer location for the impacted, adjacent and rear cylinders are compared for the two calculations of (average zone dimension of $0.286 \mathrm{~cm}$ and $0.211 \mathrm{~cm}$ ) and data in Figure 7 for
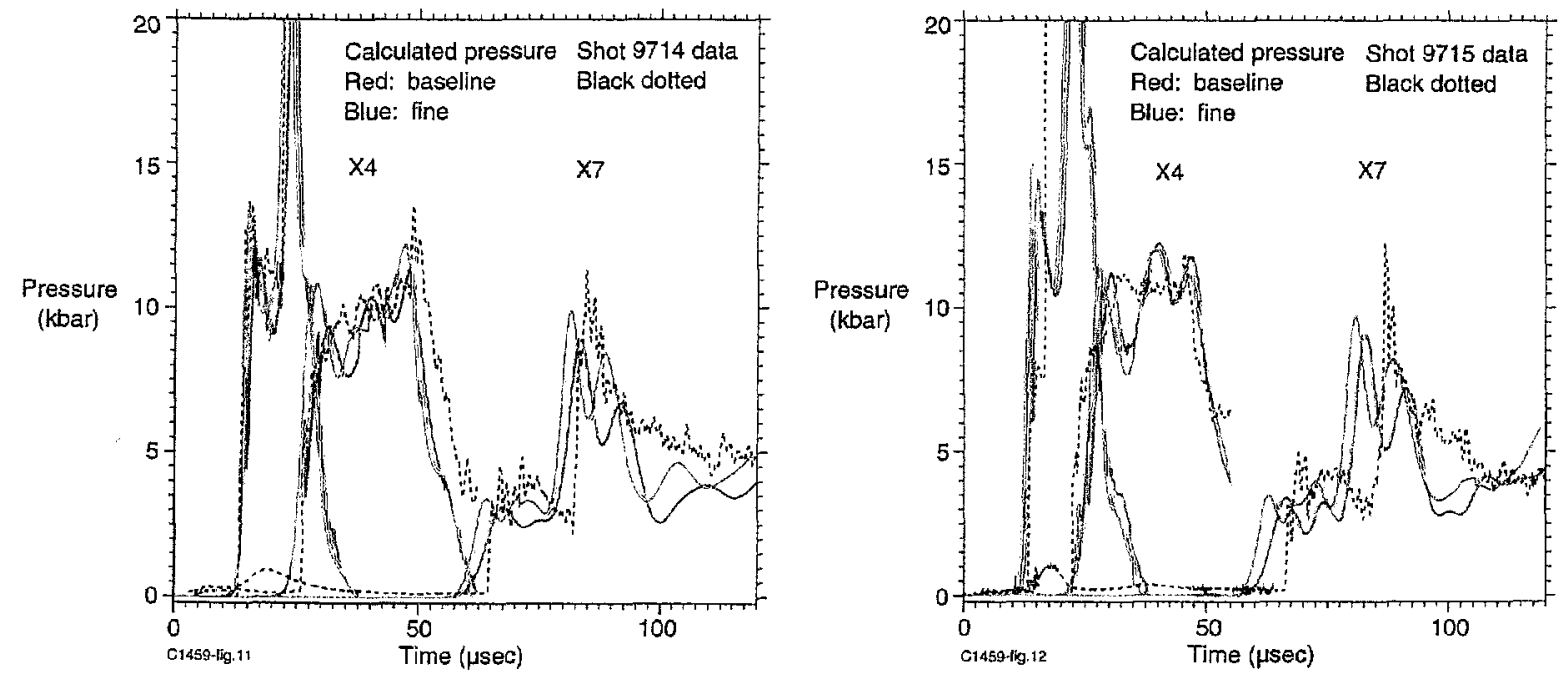

Fig. 7. Comparison of calculated and measured middle pinducer profiles for shots 9714 and 9715. One set of profiles is from a baseline calculation with $0.6 \mathrm{~cm}$ inter-cylinder spacing with an average zone dimension of $0.286 \mathrm{~cm}$, the other from a finer zoned calculation with an average zone dimension of $0.211 \mathrm{~cm}$. The impacted and adjacent cylinder pressure profiles are cut off after the first peak. The adjacent and rear cylinder profiles have been multiplied by constant factors to make their amplitude more comparable to that from the impacted cylinder. 
shot 9714 and 9715 . For the impacted cylinders there is hardly any difference between the two calculations. For the adjacent cylinder, there is some difference, but it is small. For the rear cylinder there is some difference in amplitude and some in timing, but again the differences are not substantial. In view of the large zoning dependence reported earlier, we are near convergence with respect to zone size.

\section{SUMMARY AND CONCLUSIONS}

We have made comparisons between ALE3D modeling and data from three experiments, carried out under the PLATE program, that were designed to measure quantities that can be directly compared with modeling calculations. In our modeling, several techniques are used to concentrate zoning at places of interest. We have also obtained new calibrations for the pressure gages used in the experiments.

The primary objective of the experiments was to measure pressure profiles at several locations in the impacted water filled cylinders and those adjacent to it. We have compared the measured and calculated pressure profiles at two different locations in the impacted cylinder and its closest neighbors for all three shots which produced data. The amplitude of the calculated profiles is in excellent agreement with measurements in all cases. In a couple of cases, the timing of the calculated profile differs from the measurement by about ten microseconds. Overall, the agreement between calculations and measurements is remarkable. This agreement gives confidence in our modeling calculations.

We have carried out more refined calculations for two shots and find little dependence in the calculated pressure profiles, suggesting that we are near convergence with respect to zone size.

Overall, the primary objective of the PLATE experiments has been achieved; we have been able to model pressure profiles measured at a couple of locations in several water filled cylinders on three shots. The remarkable agreement between calculated and measured profiles gives confidence in our ability to model complex experiments.

Acknowledgement-Rich Couch of LLNL provided many suggestions in using ALE3D and added tracer point features to the code that facilitated the calculations. Matt Fischer and Sue Webb of LLNL designed the targets used in the experiments. Dick Hayami of the University of Alabama at Huntsville had overall responsibility for the experiments under the guidance of Scott Howard and Mike Cole of the U.S. Army Missile Command. Jacques Charest of Dynasen, Inc., produced the new pressure gage calibrations that made the comparisons with code calculations meaningful.

This work was supported by the U.S. DOE under contract W-7405-Eng-48, the BMDO under DSWA MIPR DGAM 84030 and the OSD under IACRO DVAL80004. 


\section{REFERENCES}

[1] Lucht RA, Charest JA. Calibration and use of a rugged new piezoresistive pressure transducer. In S.C. Schmidt and W.C. Tao editors, Shock Compression of Condensed Matter. Proc. of the American Physical Society Topical Conference held in 1995, 1996: p 1041-1044.

[2] Ginsberg MJ, Asay BW. Commercial carbon composition resisters as dynamic stress gages in difficult environments. Rev. Sci. Inst., 1991; 62: 2218-2227.

[3] Wilson WH, Holloway DC, Bjarnholt G. Measurement of pressure loadings from explosively loaded boreholes used (sic) expendable piezoresistive transducers. In Techniques and Theory of Stress Wave Measurements for Shock Wave Applications. ASME, New York, 1987: p 97-108. 